\title{
Assignment of values for a set of in-house developed verification masses using an inter laboratory comparison approach
}

\author{
L. Joseph*, A. Velayudhan, and C.V. Muraleedharan \\ Sree Chitra Tirunal Institute for Medical Sciences and Technology, Trivandrum, India
}

Received: 30 September 2010 / Accepted: 4 January 2011

\begin{abstract}
Traceable measurements are a mandate for the reliability of test results. Although periodic calibration activities ensure traceability, in between verifications are essential to maintain the consistency of results. In analytical laboratories, electronic balances are widely used for quantity estimations. Owing to the inherent instability problems associated with semiconductor strain gauges and performance degradation on aging, they need to be regularly controlled using verification mass. But traceable and calibrated masses are expensive for routine use in laboratories. A mass set is designed, prepared in-house and mass values with uncertainty are assigned to undergo such verification. Steps employed to assign the mass values based on the principle of inter laboratory comparison (ILC) are reported. Reference value and corresponding uncertainty estimations are determined by performing an ILC in five different laboratories. The measurement results with uncertainty from each laboratory were received for individual masses. These results were analyzed for consistency and the values along with the weighted uncertainties were assigned.
\end{abstract}

Keywords: Verification mass; inter laboratory comparison; electronic balance; traceable measurement

\section{Nomenclature}

Notation Nomenclature

$m$

j

$y_{j}$

$n$

$i$

$x_{i}$

$\bar{x}$

$u_{i}$

$k$

$\sigma_{m-1}$

$R_{B}$

$S_{\text {ext }}$

$S_{\text {int }}$

$w_{i}$

$x_{w}$

$x_{\text {ref }}$

$U\left(x_{\text {ref }}\right)$

$S^{2}$

$\alpha$

$w_{i}^{\prime}$

$s_{w}$

$s_{u}$

Coverage factor

of measurements

Birge's ratio

Weighted mean

Variance
Total number of measurements

from a single laboratory

Single measurement, 1 to $m$

Single measurement

from an individual laboratory

Total number of laboratories involved

Individual laboratory, 1 to $n$

Mean mass value reported

by $i$ th laboratory

Unweighted mean estimated

from $n$ number of $x_{i}$

Standard uncertainty reported

by $i$ th laboratory $(k=1)$

Standard deviation of $m$ number

External uncertainty

Internal uncertainty

Weightage factor

Reference mass to be assigned

Uncertainty to be assigned

Level of trust in relative mass values

Proposed weighting factor

Uncertainty of the "adjusted" weighted mean

Uncertainty of the unweighted mean, $\bar{x}$

\section{Introduction}

For a variety of reasons, electronic balances can either malfunction or their outputs drift during routine use [1]. Drift in sensitivity or linearity, transfer of location of use, changes in environmental conditions etc are the most commonly identified sources of variance that occur during the normal course of measurements. Sensitivity errors can be caused by temperature drift, aging or due to off-center error. Aging of the load cell and electronic instrumentation, or overloading of the balance can be the reasons for drift in linearity. Shifting of the balance to a new location can introduce errors due to changes in acceleration due to gravity and shift in the vertical direction of the measurement axis. Variations in temperature and humidity at the location of measurements also can introduce errors in the results.

Such errors may significantly contribute to the total uncertainty of a measurement unless they are timely identified and corrected. Furthermore they may determine the acceptability of a product or the outcome of a test. Hence it is important to have procedures for assuring the quality of weighing results $[2,3]$. Verification using suitable verification mass is a simple way to assure the proper performance of an electronic balance [4]. The creation of a control chart based on the verification mass readings would help identifying drifts and similar nonconformities and could constitute inputs for corrective actions.

Since traceable mass standards are expensive, many laboratories do not practice these verifications. Therefore balance performance is only verified during calibration,

* Correspondence: leenaj@sctimst.ac.in 
Table 1. Design parameters for the verification mass with property specifications.

\begin{tabular}{ll}
\hline Shape & Cylindrical \\
Raw material & Stainless steel (SS 304) \\
Nominal mass & $1 \mathrm{~g}$ \\
Accuracy & Class M2 \\
Nominal dimensions & $6 \mathrm{~mm}(\varphi) \times 7.4 \mathrm{~mm}$ (height) \\
Surface finish & Less than $0.5 \mu \mathrm{m}$ \\
Appearance of surface & Smooth without defects and surface porosities \\
\hline
\end{tabular}

which happens only once or twice a year. Therefore there is a need for a cost effective option for routine verification of electronic balances, especially when a large number of balances are deployed in a location. Use of in house verification masses could be one solution for this problem. Although many laboratories may have facilities for producing such verification masses, assigning appropriate mass values would require the help of mass calibration agencies, which makes the process costlier. Inter laboratory comparison is an established technique employed for value assignment [5]. A method based on conventional inter laboratory comparison approach is presented here for assigning values for in house generated verification masses.

\section{Materials and methods}

\subsection{Design and characterization}

Design and preparation of masses were carried out based on International Organization of Legal Metrology (OIML) R111-1 which describes the characteristics for weights in different accuracy classes [6]. The design specifications are provided in Table 1.

Stainless steel SS 304 was selected for the fabrication of the masses. Table 2 provides the elemental composition of SS 304 [7]. Rods of raw material were cut and machined using computer numerical controlled (CNC) turning/milling operations to get a mass of $1 \mathrm{~g}$ nominal value in cylindrical shape with a knob (Fig. 1). A total of 20 masses identified as M1 to M20 were fabricated.

\subsection{Polishing}

Hand lapping using 600 mesh silicone carbide papers was carried out on the samples to trim their mass values within the class M2 accuracy range $(1 \mathrm{~g} \pm 0.003 \mathrm{~g})$. Subsequently, a three stage polishing (viz. wet cut down, dry polishing and ball burnishing) was carried out in a centrifugal tumbling machine (M/s. Dreher GmbH, Germany) and polishing media (M/s. Dreher GmbH, Germany) to improve the surface finish and to smoothen the edges.

\subsubsection{Wet cut down}

Samples are mixed with media consisting of $0.6 \mathrm{~L}$ of plastic cones (PC-12), 0.6 L of ceramic chips (DG-4) and $100 \mathrm{~mL}$
Table 2. American Society for Metals (ASM) specifications for stainless steel SS 304 [7].

\begin{tabular}{cc}
\hline Parameter & Characteristic property \\
\hline Composition & \\
\hline Carbon & $0.08 \%$ (maximum) \\
Manganese & $2.00 \%$ (maximum) \\
Phosphorus & $0.045 \%$ (maximum) \\
Sulfur & $0.030 \%$ (maximum) \\
Silicon & $0.75 \%$ (maximum) \\
Chromium & $18.00 \%-20.00 \%$ \\
Nickel & $8.00 \%-12.00 \%$ \\
Nitrogen & $0.10 \%$ (maximum) \\
Iron & Balance \\
\hline Strength, yield & $215 \mathrm{MPa}$ at $0.2 \%$ offset \\
\hline Density & $8 \mathrm{~g} / \mathrm{cm}^{3}$ \\
\hline
\end{tabular}

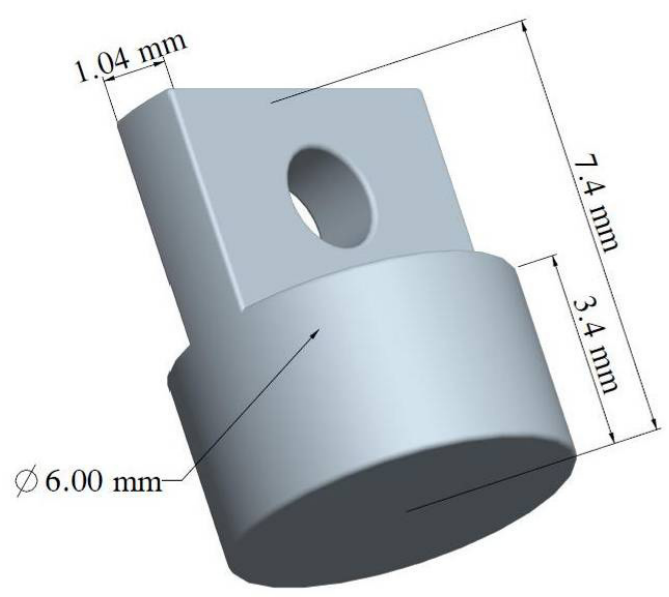

Fig. 1. Physical model of the verification mass.

of A60 polishing compound. The mixture is loaded in the centrifugal tumbling machine with $75 \%$ water in a $2.5 \mathrm{~L}$ barrel and processed for $6 \mathrm{~h}$ at $220 \mathrm{rpm}$.

\subsubsection{Dry polishing}

The polishing media used at this stage is a mixture of $2 \mathrm{~L}$ of treated wooden granules (TSG-2), $36 \mathrm{~mL}$ deburring paste (TSP-2) and $30 \mathrm{~mL}$ silicon carbide powder with 600 mesh size. Dry polishing is carried out for $2 \mathrm{~h}$ at a 220 rpm speed. 


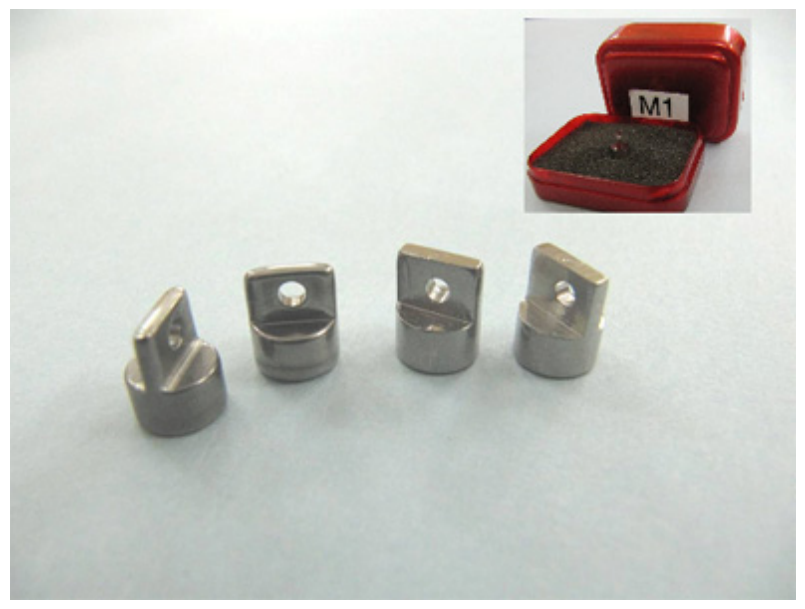

Fig. 2. Set of polished mass samples. Inset shows the standard packaging provided.

\subsubsection{Ball burnishing}

Polishing with $2 \mathrm{~L}$ of wooden granules (TPG 1), $25 \mathrm{~mL}$ of polishing powder, $100 \mathrm{~mL}$ of $3 \mathrm{~mm} \varphi$ steel balls (M/s. Inox Corporation, USA) and $20 \mathrm{~mL}$ of dispersing fluid (SFF) is carried out at $210 \mathrm{rpm}$ for $3 \mathrm{~h}$.

Standard ultrasonic cleaning was carried out to get the samples, free of contaminants and grease. The samples were dried in a conventional hot air oven and packed in individual containers (Fig. 2).

\subsection{Visual inspection}

Visual inspection was carried out with stereo microscope SMZ 800 (M/s. NIKON Corporation, Japan) under 100 X to $150 \mathrm{X}$ magnification to assess the smoothness of surface and to detect any major defects on the samples.

\subsection{Surface finish}

The surface finish was characterized using a non contact profilometer, Talysurf CLI 1000 (M/s. Taylor Hobson, UK). Measurement was carried out using chromatic length aberration (CLA) confocal gauge with a step height repeatability of $2 \mathrm{~nm}$. Line profiles in the range of $300 \mu \mathrm{m}$ with $0.5 \mu \mathrm{m}$ spacing were taken at random locations.

\subsection{Density measurement}

Density measurement was carried out using the density determination kit and the electronic balance ME $414 \mathrm{~S}$ (M/s. Sartorius, Germany) with $0.1 \mathrm{mg}$ resolution.

\subsection{Inter laboratory comparison}

Mass measurements for all the 20 masses were carried out in an inter laboratory comparison method involving five
Table 3. Details of balances participated in ILC.

\begin{tabular}{llll}
\hline Balance Id & Traceability & $\begin{array}{l}\text { Resolution } \\
(\mathrm{mg})\end{array}$ & $\begin{array}{l}\text { Linearity } \\
(\mathrm{mg})\end{array}$ \\
\hline B1 & 0.1 & \pm 0.2 \\
B2 & \multirow{2}{*}{ NPL, New Delhi } & 0.1 & \pm 0.2 \\
B3 & & 0.01 & \pm 0.15 \\
\hline B4 & DKD, Germany & 0.01 & \pm 0.1 \\
B5 & & 0.1 & \pm 0.3 \\
\hline
\end{tabular}

laboratory balances B1 to B5 [8]. Details of the balances used in the study are provided in Table 3. Six independent measurements were taken for each mass sample by four different operators employing five different electronic balances. Since all balances were located in a single campus, it was possible to get the measurements carried out by each operator in all balances, leading to a total of $120(6 \times 4 \times 5)$ measurements for each mass sample. All operators involved were experienced and trained in this institution accredited by Le Comité Français d'Accréditation (COFRAC) of France for biomaterial testing. All laboratory balances employed were calibrated and traceable to National Physical Laboratory (NPL), India or Deutschen Kalibrierdienst (DKD), Germany.

Individual measurement reports were obtained for all the mass samples from each laboratory as $x_{i} \pm u_{i}$. Standard measurement uncertainties from following elements were combined while estimating the uncertainty $u_{i}$.

- Repeatability associated with measurements $y_{j}$ carried out by four operators, with six replication each $(m=$ $6 \times 4=24$ ).

- Uncertainty in the calibration of the balance.

- Resolution of the balance.

- Linearity of the balance.

Combined standard uncertainty in the mass measurement is given by

$$
u_{i}=\sqrt{u_{1}^{2}+u_{2}^{2}+u_{3}^{2}+u_{4}^{2}}
$$

where:

$$
\begin{gathered}
u_{1}=\frac{\sigma_{m-1}}{\sqrt{m}}, \\
\sigma_{m-1}=\sqrt{\frac{\sum_{j=1}^{m}\left(y_{j}-x_{i}\right)^{2}}{m-1}} \\
x_{i}=\sum_{j=1}^{m} \frac{y_{j}}{m}
\end{gathered}
$$

$u_{2}=$ Uncertainty in the balance calibration $/ 2$

$u_{3}=$ Resolution of balance $/ 2 \sqrt{ } 3$

$u_{4}=$ Linearity of balance $/ \sqrt{ } 3$.

\subsection{Assigning mass values}

The results obtained from individual laboratories $x_{i} \pm u_{i}$, were analyzed based on the strategy proposed by Pommé and Spasova [9]. The assignment of the reference mass value is a three stage process as described in Figure 3. 
Value assignment for verification mass by ILC

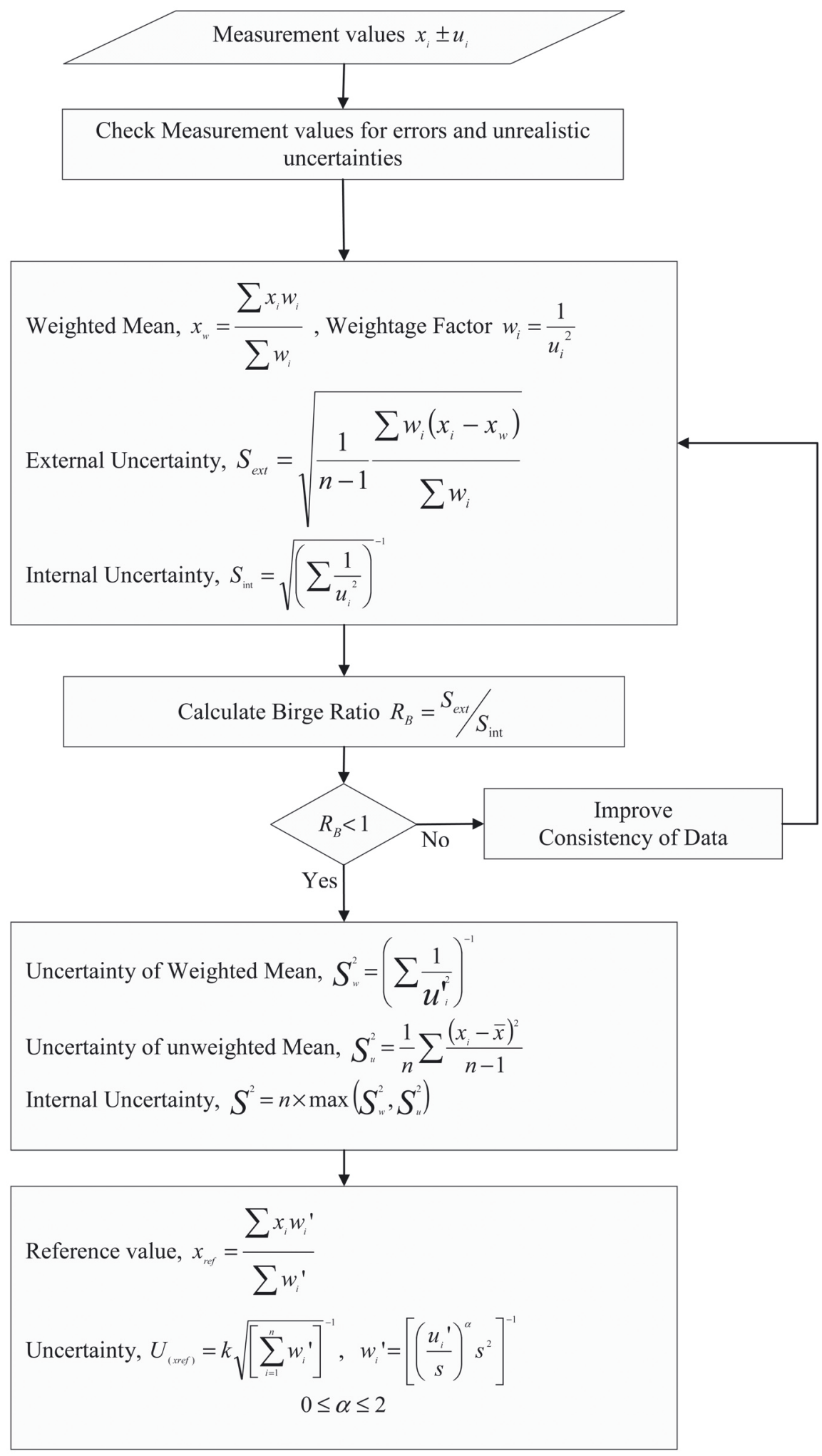

Fig. 3. Steps in the process of assigning mass value. 


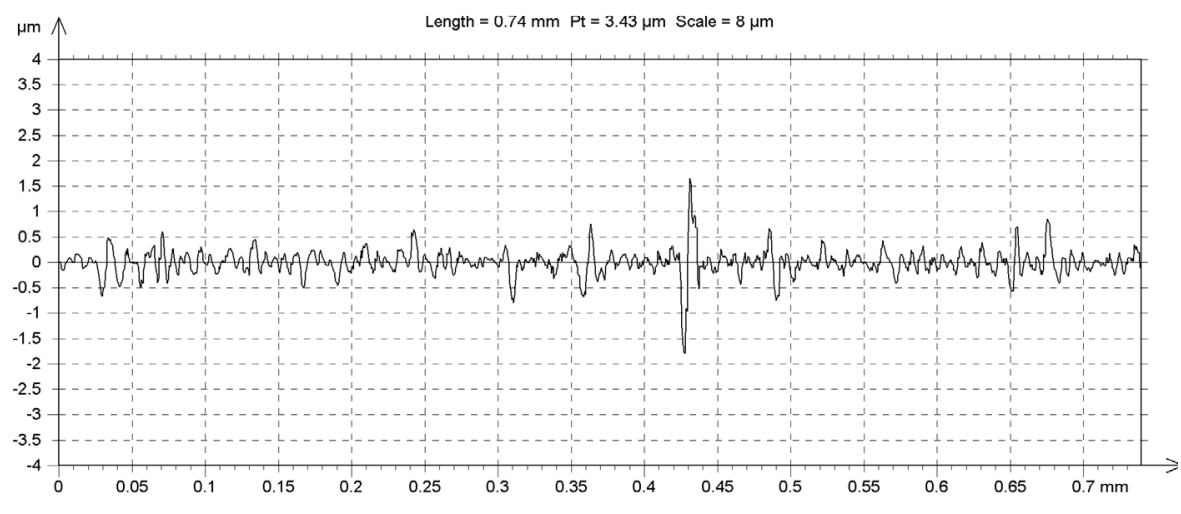

Fig. 4. Indicative line profile of finished mass surfaces measured using CLA gauge. Average surface roughness observed for all the inspected samples were less than $0.5 \mu \mathrm{m}$ Ra.

1. Identification and correction of errors and unrealistic uncertainties.

2. Detection of discrepancies and making the data consistent.

3. Assignment of reference value along with its associated uncertainty.

In the first step the data from individual laboratories is scrutinized for possible errors. The uncertainty values reported from different laboratories were realistic and did not seem to have substantial errors and hence no action was required with respect to the first step.

The second step involves the assessment of the consistency of the data and if the data scatter is larger than expected, corrective measures must be taken to bring the data to the required level of consistency. This is achieved by calculating the Birge ratio $\left(R_{B}\right)$.

$$
R_{B}=\frac{S_{\text {ext }}}{S_{\text {int }}}
$$

where

$$
\begin{aligned}
S_{\text {ext }} & =\sqrt{\frac{1}{n-1} \frac{\sum_{i=1}^{n} w_{i}\left(x_{i}-x_{w}\right)^{2}}{\sum_{i=1}^{n} w_{i}}} \\
S_{\mathrm{int}} & =\sqrt{\left(\sum_{i=1}^{n} \frac{1}{u_{i}^{2}}\right)^{-1}} \\
w_{i} & =\frac{1}{u_{i}^{2}}, x_{w}=\sum_{i=1}^{n} \frac{x_{i} w_{i}}{\sum_{i=1}^{n} w_{i}} .
\end{aligned}
$$

If $R_{B}<1$, it can be inferred that the data is consistent and hence no further efforts are required for improving the consistency of the data set. If $R_{B} \geqslant 1$, the consistency could be improved by

- looking for outliers and eliminating them with proper justification.

- increasing the uncertainties $\left(u_{i}\right)$ with a suitable constant " $a$ " as $u_{i}^{\prime}=\sqrt{u_{i}^{2}+a^{2}}$ until the values of $R_{B}$ is brought down to less than 1 .
In the third step, the reference value $x_{\text {ref }}$ and uncertainty $U\left(x_{\text {ref }}\right)$ are estimated as,

$$
\begin{gathered}
x_{\mathrm{ref}}=\sum_{i=1}^{n}\left[\frac{x_{i} w_{i}^{\prime}}{\sum_{i=1}^{n} w_{i}^{\prime}}\right] \\
U\left(x_{\mathrm{ref}}\right)=k \sqrt{\left[\sum_{i=1}^{n} w_{i}^{\prime}\right]^{-1}} .
\end{gathered}
$$

where,

$$
w_{i}^{\prime}=\left[\left(\frac{u_{i}}{S}\right)^{\alpha} S^{2}\right]^{-1}
$$

Variance, $S^{2}=$ maximum of $\left\{s_{w}^{2}, s_{u}^{2}\right\} \times n$, where

$$
\begin{gathered}
s_{w}^{2}=\left(\sum_{i=1}^{n} \frac{1}{u_{i}^{2}}\right)^{-1} \\
s_{u}^{2}=\frac{1}{n} \frac{\sum\left(x_{i}-\bar{x}\right)^{2}}{n-1}
\end{gathered}
$$

The coverage factor $k$ is chosen as 1.96 for a confidence level of $95 \%$ and the variable $\alpha$, which signifies the level of trust in relative weights, can vary between 0 and 2 .

\section{Results and discussion}

In the visual inspection, the mass surfaces were found to be smooth and free of any detectable irregularities. They did not have any sharp edges or corners likely to cause deterioration nor any pronounced imperfections likely to cause deposits (i.e. of dust) on their surface. Density was estimated to be $7.94 \mathrm{~g} / \mathrm{cm}^{3} \pm 0.004 \mathrm{~g} / \mathrm{cm}^{3}$ as mean value \pm 1 standard deviation, which is the density reported for SS 304. The average surface roughness was estimated to be $0.29 \mu \mathrm{m} \pm 0.11 \mu \mathrm{m} R a$ (mean value \pm 1 standard deviation). A representative line profile is shown in Figure 4. Mean values $\left(x_{i}\right)$ and the corresponding measurement uncertainty $\left(u_{i}\right)$ for individual masses reported by ILC participants is provided in Table 4. 
Table 4. Mean values $x_{i}$ and measurement uncertainties $u_{i}$ reported for the masses M1 to M20 by laboratories B1 to B5.

\begin{tabular}{|c|c|c|c|c|c|c|c|c|c|c|}
\hline \multirow{3}{*}{ Mass ID } & \multicolumn{2}{|c|}{ B1 } & \multicolumn{2}{|c|}{ B2 } & \multicolumn{2}{|c|}{ B3 } & \multicolumn{2}{|c|}{ B4 } & \multicolumn{2}{|c|}{ B5 } \\
\hline & $x_{i}$ & $u_{i}$ & $x_{i}$ & $u_{i}$ & $x_{i}$ & $u_{i}$ & $x_{i}$ & $u_{i}$ & $x_{i}$ & $u_{i}$ \\
\hline & \multicolumn{10}{|c|}{ (g) } \\
\hline M1 & 1.0003 & 0.00024 & 1.0003 & 0.00024 & 1.0003 & 0.00017 & 1.00030 & 0.00012 & 1.00030 & 0.00035 \\
\hline M2 & 1.0012 & 0.00024 & 1.0012 & 0.00024 & 1.0012 & 0.00017 & 1.00117 & 0.00012 & 1.00119 & 0.00035 \\
\hline M3 & 1.0008 & 0.00024 & 1.0007 & 0.00024 & 1.0008 & 0.00017 & 1.00076 & 0.00012 & 1.00078 & 0.00035 \\
\hline M4 & 1.0019 & 0.00024 & 1.0019 & 0.00024 & 1.0019 & 0.00017 & 1.00191 & 0.00012 & 1.00193 & 0.00035 \\
\hline M5 & 0.9997 & 0.00024 & 0.9996 & 0.00024 & 0.9997 & 0.00017 & 0.99967 & 0.00012 & 0.99967 & 0.00035 \\
\hline M6 & 0.9989 & 0.00024 & 0.9988 & 0.00024 & 0.9989 & 0.00017 & 0.99886 & 0.00012 & 0.99887 & 0.00035 \\
\hline M7 & 1.0008 & 0.00024 & 1.0008 & 0.00024 & 1.0008 & 0.00017 & 1.00083 & 0.00012 & 1.00083 & 0.00035 \\
\hline M8 & 0.9949 & 0.00024 & 0.9949 & 0.00024 & 0.9949 & 0.00017 & 0.99490 & 0.00012 & 0.99489 & 0.00035 \\
\hline M9 & 0.9986 & 0.00024 & 0.9986 & 0.00024 & 0.9987 & 0.00017 & 0.99865 & 0.00012 & 0.99864 & 0.00035 \\
\hline M10 & 0.9997 & 0.00024 & 0.9997 & 0.00024 & 0.9997 & 0.00017 & 0.99975 & 0.00012 & 0.99973 & 0.00035 \\
\hline M11 & 0.9989 & 0.00024 & 0.9989 & 0.00024 & 0.9989 & 0.00017 & 0.99890 & 0.00012 & 0.99892 & 0.00035 \\
\hline M12 & 1.0008 & 0.00024 & 1.0008 & 0.00024 & 1.0008 & 0.00017 & 1.00077 & 0.00012 & 1.00078 & 0.00035 \\
\hline M13 & 1.0011 & 0.00024 & 1.0011 & 0.00024 & 1.0011 & 0.00017 & 1.00114 & 0.00012 & 1.00114 & 0.00035 \\
\hline M14 & 1.0000 & 0.00024 & 1.0001 & 0.00024 & 1.0000 & 0.00017 & 1.00004 & 0.00012 & 1.00005 & 0.00035 \\
\hline M15 & 0.9995 & 0.00024 & 0.9994 & 0.00024 & 0.9995 & 0.00017 & 0.99945 & 0.00012 & 0.99945 & 0.00035 \\
\hline M16 & 0.9794 & 0.00024 & 0.9794 & 0.00024 & 0.9794 & 0.00017 & 0.97936 & 0.00012 & 0.97939 & 0.00035 \\
\hline M17 & 1.0005 & 0.00024 & 1.0005 & 0.00024 & 1.0005 & 0.00017 & 1.00046 & 0.00012 & 1.00047 & 0.00035 \\
\hline M18 & 1.0006 & 0.00024 & 1.0006 & 0.00024 & 1.0006 & 0.00017 & 1.00058 & 0.00012 & 1.00058 & 0.00035 \\
\hline M19 & 0.9949 & 0.00024 & 0.9949 & 0.00024 & 0.9949 & 0.00017 & 0.99489 & 0.00012 & 0.99489 & 0.00035 \\
\hline M20 & 0.9980 & 0.00025 & 0.9980 & 0.00024 & 0.9980 & 0.00017 & 0.99803 & 0.00012 & 0.99805 & 0.00035 \\
\hline
\end{tabular}

Table 5. Results of Birge ratio estimation for individual masses.

\begin{tabular}{lc}
\hline Mass ID & $R_{B}$ \\
\hline M1 & 0.116 \\
M2 & 0.127 \\
M3 & 0.126 \\
M4 & 0.108 \\
M5 & 0.117 \\
M6 & 0.107 \\
M7 & 0.090 \\
M8 & 0.071 \\
M9 & 0.097 \\
M10 & 0.062 \\
M11 & 0.148 \\
M12 & 0.146 \\
M13 & 0.174 \\
M14 & 0.065 \\
M15 & 0.076 \\
M16 & 0.111 \\
M17 & 0.110 \\
M18 & 0.105 \\
M19 & 0.115 \\
M20 & 0.090 \\
\hline
\end{tabular}

The estimated values of Birges ratio, $R_{B}$, based in equation (2) for different data sets are provided in Table 5. It can be noted that the $R_{B}$ values are less than unity indicating that data sets are consistent. The data scatter are not larger than those expected from declared uncertainties, and no further modifications are required to improve the consistency of these data sets. In view of this, $u_{i}^{\prime}=u_{i}$ and the level of trust in relative mass values, $\alpha$ can be chosen as 2 for estimating the fully weighted mean $x_{\text {ref }}$ and the corresponding uncertainty values, $U\left(x_{\text {ref }}\right)$. This
Table 6. Estimation of reference mass value, $x_{\text {ref }}$ and associated uncertainty $U\left(x_{\text {ref }}\right)$ for mass $\mathrm{M} 1$ as a representative model.

\begin{tabular}{ccc}
\hline Sl. No & Parameter & Estimate \\
\hline 1 & $\begin{array}{c}\text { Weighted average } \\
\left(x_{w}\right)=\sum\left(x_{i} w_{i}\right) / \sum w_{i}\end{array}$ & $1.000302 \mathrm{~g}$ \\
\hline 2 & $\begin{array}{c}\text { External uncertainty } \\
\left(S_{\text {ext }}\right)=\sqrt{\frac{1}{n-1} \frac{\sum w_{i}\left(x_{i}-x_{w}\right)^{2}}{\sum w_{i}}}\end{array}$ & $0.000005 \mathrm{~g}$ \\
\hline 3 & $\begin{array}{c}\text { Internal uncertainty } \\
\left(S_{\text {int }}\right)=\sqrt{\left(\sum 1 / u_{i}^{2}\right)^{-1}}\end{array}$ & $0.000042 \mathrm{~g}$ \\
\hline 4 & Birge ratio $\left(R_{B}\right)=\frac{S_{\text {ext }}}{S_{\text {int }}}$ & 0.116114 \\
\hline 5 & Reference value $x_{\text {ref }}=x_{w}$ & $1.0003 \mathrm{~g}$ \\
\hline 6 & Uncertainty of the weighted \\
& average $U\left(x_{\text {ref }}\right)$ & $0.0821 \mathrm{mg}$ \\
\hline
\end{tabular}

level of consistency achieved in the data sets is due to the following.

(a) All electronic balances employed for this ILC are calibrated using the same reference mass standard.

(b) The same team of trained operators carried out the measurements.

Most often, in such ILC based data collections, a certain level of inconsistency should be expected and efforts to improve the consistency as described in Section 2.7 will have to be made. In the case of inconsistent data sets, if the analysis does not address the correction of uncertainty to bring the Birge ratio below unity, the estimate of the mean value $\left(x_{\text {ref }}\right)$ will still be realistic, but the uncertainty associated with $x_{\text {ref }}$ will be underestimated [9].

$x_{\text {ref }}$ and $U\left(x_{\text {ref }}\right)$ values were estimated based in equations (3) and (4). A sample calculation for mass M1 is provided in Table 6 and the final values assigned for the 
Table 7. Assigned reference value with uncertainty for all in house reference masses.

\begin{tabular}{lcc}
\hline Mass ID & $x_{\text {ref }}(\mathrm{g})$ & $\pm U\left(x_{\text {ref }}\right)(\mathrm{mg})$ \\
\hline M1 & 1.0003 & 0.0821 \\
M2 & 1.0012 & 0.0820 \\
M3 & 1.0008 & 0.0821 \\
M4 & 1.0019 & 0.0821 \\
M5 & 0.9997 & 0.0821 \\
M6 & 0.9989 & 0.0821 \\
M7 & 1.0008 & 0.0821 \\
M8 & 0.9949 & 0.0821 \\
M9 & 0.9986 & 0.0821 \\
M10 & 0.9997 & 0.0822 \\
M11 & 0.9989 & 0.0821 \\
M12 & 1.0008 & 0.0821 \\
M13 & 1.0011 & 0.0821 \\
M14 & 1.0000 & 0.0821 \\
M15 & 0.9995 & 0.0820 \\
M16 & 0.9794 & 0.0821 \\
M17 & 1.0005 & 0.0821 \\
M18 & 1.0006 & 0.0821 \\
M19 & 0.9949 & 0.0821 \\
M20 & 0.9980 & 0.0821 \\
\hline
\end{tabular}

20 different mass samples are provided in Table 7. Of the twenty samples prepared initially, seventeen of them were obtained within the accuracy limits of OIML class M2 weights. The estimated uncertainty levels were found to be better than $\pm 0.1 \mathrm{mg}$ which is acceptable for a mass with accuracy of $\pm 3 \mathrm{mg}$. This indicates that the technique could be employed for masses with better accuracy levels also. These verification masses are being regularly used in more than fifteen laboratories in the institute.

The proposed method for assigning mass values for in house developed verification masses could be extended to many value assignment cases, where the experimental data is limited. This ILC based approach reduces the cost of value assignment and provides a means for establishing the traceability through available in-house metrology facilities.
Acknowledgements. The authors wish to acknowledge the support of the divisions of Precision Fabrication Facility, Device Testing Laboratory of the Institute for their invaluable contributions in the fabrication and characterization of the materials.

\section{References}

1. National Association of Testing Authorities (NATA), NATA Technical Note 13: User checks and maintenance of laboratory Balances (NATA, Australia, 2007)

2. Xiaohua Lu, Hongmei Li, Fanmin Meng, Jie Ji, The establishment of a quality evaluation system of reference materials, Accred. Qual. Assur. 14, 329 (2009)

3. C.M. Sutton, Assuring the Quality of Weighing results, Measurement Standards Laboratory (MSL) Technical Guide 12 (MSL, New Zealand, 2006)

4. A.G. Gonzalez, M.A. Heerrador, The assessment of electronic balances for accuracy of mass measurements in the analytical laboratory, Accred. Qual. Assur. 12, 21 (2007)

5. Committee on reference materials (REMCO), International Organization for standardization (ISO) Guide 34, General requirements for the competence of reference material producers (ISO, Switzerland, 2009)

6. International Organization of Legal Metrology (OIML) technical committee $\mathrm{TC}$ 9/SC 3 Weights, International Recommendation-OIML R 111-1: Weights of classes E1, E2, F1, F2, M1, M1-2, M2, M2-3 and M3 Part 1: Metrological and technical requirements (OIML, Paris, 2004)

7. D. Benjamin, Metals Handbook: Properties and Selection: Steels, Tool materials and special purpose metals (American Society for Metals (ASM) International, USA, 1980), Vol. 3, pp. 5-34

8. Committee on reference materials (REMCO), ISO Guide 35, Reference materials, General and statistical principles for certification (ISO, Switzerland, 2006)

9. S. Pommé, Y. Spasova, A practical procedure for assigning a reference value and uncertainty in the frame of an Interlaboratory comparison, Accred. Qual. Assur. 13, 83 (2008) 